\title{
Author Index Volume 35 (2013)
}

The issue number is given in front of the pagination

Abbate, C., see Arosio, B. (4) 669-674

Abner, E.L., see Kryscio, R.J. (4) 823-832

Abramsson, A., see Daborg, J. (1) 51-57

Acharya, N.K., E.C. Levin, P.M. Clifford, M. Han, R. Tourtellotte, D. Chamberlain, M. Pollaro, N.J. Coretti, M.C. Kosciuk, E.P. Nagele, C. DeMarshall, T. Freeman, Y. Shi, C. Guan, C.H. Macphee, R.L. Wilensky and R.G. Nagele, Diabetes and Hypercholesterolemia Increase Blood-Brain Barrier Permeability and Brain Amyloid Deposition: Beneficial Effects of the LpPLA2 Inhibitor Darapladib (1) 179-198

Admiraal-Behloul, F., see van der Vlies, A.E. (1) 169178

Adorni, F., see Di Santo, S.G. (2) 349-361

Aguayo, L.G., see Zurita, M.P. (3) 463-473

Akatsu, H., see Wen, Y. (2) 387-394

Akazawa, K., see Wen, Y. (2) 387-394

Andreasson, U., see Daborg, J. (1) 51-57

Andrews, R.D., see Mosconi, L. (3) 509-524

Andrieux, A., see Nissou, M.-F. (3) 553-564

Antonell, A., see Arenaza-Urquijo, E.M. (4) 715-726

Antonenko, D., see Thurm, F. (3) 575-587

Antúnez, C., see Martinez-Mir, A. (2) 403-412

Arbuckle, M., see Claxton, A. (4) 789-797

Arenaza-Urquijo, E.M., J.-L. Molinuevo, R. SalaLlonch, C. Solé-Padullés, M. Balasa, B. Bosch, J. Olives, A. Antonell, A. Lladó, R. Sánchez-Valle, L. Rami and D. Bartrés-Faz, Cognitive Reserve Proxies Relate to Gray Matter Loss in Cognitively Healthy Elderly with Abnormal Cerebrospinal Fluid Amyloid- $\beta$ Levels (4) 715-726

Arosio, B., C. Abbate, D. Galimberti, P.D. Rossi, S. Inglese, C. Fenoglio, E. Ridolfi, C. Gussago, M. Casati, E. Tedone, E. Ferri, M. Serpente, E. Scarpini and D. Mari, GRN Thr272fs Clinical Heterogeneity: A Case with Atypical Late Onset Presenting with a Dementia with Lewy Bodies Phenotype (4) 669-674

Artjomova, S., see Schmidt, C. (3) 451-454

Aso, E., S. Juvés, R. Maldonado and I. Ferrer, $\mathrm{CB}_{2}$ Cannabinoid Receptor Agonist Ameliorates
Alzheimer-Like Phenotype in A $\beta P P / P S 1$ Mice (4) $847-858$

Atifi, M.E., see Nissou, M.-F. (3) 553-564

Backes, W.H., see Burgmans, S. (4) 859-873

Baker, L.D., see Claxton, A. (4) 789-797

Balasa, M., see Arenaza-Urquijo, E.M. (4) 715-726

Bancher, C., see Benke, T. (2) 247-252

Banks, W.A., see Rhodehouse, B.C. (1) 59-66

Barber, R.C., see Hall, J.R. (2) 363-371

Barkhof, F., see van der Vlies, A.E. (1) 169-178

Barnes, J., see Scahill, R.I. (1) 199-212

Barrio, J.R., see Merrill, D.A. (1) 147-157

Bartlett, J.W., see Scahill, R.I. (1) 199-212

Bartrés-Faz, D., see Arenaza-Urquijo, E.M. (4) 715-726

Bastard, N.L., see Slaets, S. (1) 137-146

Basun, H., see Hjorth, E. (4) 697-713

Bayer, T.A., see Wirths, O. (4) 741-749

Bearden, S.E., see Rhodehouse, B.C. (1) 59-66

Beck, J., see Scahill, R.I. (1) 199-212

Benke, T., M. Delazer, G. Sanin, H. Schmidt, S. Seiler, G. Ransmayr, P. Dal-Bianco, M. Uranüs, J. Marksteiner, F. Leblhuber, P. Kapeller, C. Bancher and R. Schmidt, Cognition, Gender, and Functional Abilities in Alzheimer's Disease: How are They Related? (2) 247-252

Berger, F., see Nissou, M.-F. (3) 553-564

Berr, C., see Vercambre, M.-N. (2) 413-421

Bettayeb, K., see Hochard, A. (1) 107-120

Bhatia, S., A.M. Jenner, H. Li, K. Ruberu, A.S. Spiro, C.E. Shepherd, J.J. Kril, N. Kain, A. Don and B. Garner, Increased Apolipoprotein D Dimer Formation in Alzheimer's Disease Hippocampus is Associated with Lipid Conjugated Diene Levels (3) 475-486

Blennow, K., see Daborg, J. (1) 51-57

Blennow, K., see Shahim, P. (2) 307-312

Blennow, K., see Sjölander, A. (1) 121-127

Boada, M., see Martinez-Mir, A. (2) 403-412

Bochem, A.E., see Shahim, P. (2) 307-312

Bondareff, W., Age-Related Changes in Brain Extracellular Space Affect Processing of 
Amyloid- $\beta$ Peptides in Alzheimer's Disease (1) 1-6

Bonsi, R., see Villa, C. (3) 487-494

Bookheimer, S.Y., see Merrill, D.A. (1) 147-157

Borgne-Sanchez, A., see Hochard, A. (1) 107-120

Bosch, B., see Arenaza-Urquijo, E.M. (4) 715-726

Bosma, H., see Vermeiren, A.P.A. (2) 241-246

Brocard, J., see Nissou, M.-F. (3) 553-564

Broeckhoven, C.V., see Slaets, S. (1) 137-146

Brouwers, N., see Engelborghs, S. (1) 67-73

Bruner, E., and H.I.L. Jacobs, Alzheimer's Disease: The

Downside of a Highly Evolved Parietal Lobe? (2) 227-240

Bruno, G., see Piscopo, P. (2) 313-318

Buckles, V.D., see Shim, Y.S. (4) 799-811

Budinich, M., see Slachevsky, A. (2) 297-306

Burgmans, S., H.J. van de Haar, F.R.J. Verhey and W.H. Backes, Amyloid- $\beta$ Interacts with BloodBrain Barrier Function in Dementia: A Systematic Review (4) 859-873

Burgos, C.F., see Zurita, M.P. (3) 463-473

Burns, J.M., see Watts, A.S. (2) 253-265

Buron, N., see Hochard, A. (1) 107-120

Caffarra, P., see Mitolo, M. (1) 75-90

Caltagirone, C., see Di Santo, S.G. (2) 349-361

Campion, D., see Pottier, C. (1) 45-49

Campos-Peña, V., see Lira-De León, K.I. (2) 319-334

Cantoni, C., see Villa, C. (3) 487-494

Cao, H.-Y., see Han, G. (2) 373-386

Cappa, S., see Villa, C. (3) 487-494

Cappa, S.F., see Cerami, C. (3) 455-462

Cardoso, S., see Carvalho, C. (3) 623-635

Carvalho, C., N. Machado, P.C. Mota, S.C. Correia, S. Cardoso, R.X. Santos, M.S. Santos, C.R. Oliveira and P.I. Moreira, Type 2 Diabetic and Alzheimer's Disease Mice Present Similar Behavioral, Cognitive, and Vascular Anomalies (3) 623-635

Casarejos, M.J., J. Perucho, A. Gomez, M.P. Muñoz, M. Fernandez-Estevez, O. Sagredo, J.F. Ruiz, M. Guzman, J.G. de Yebenes and M.A. Mena, Natural Cannabinoids Improve Dopamine Neurotransmission and Tau and Amyloid Pathology in a Mouse Model of Tauopathy (3) 525-539

Casati, M., see Arosio, B. (4) 669-674

Castellani, R.J., see Perry, G. (3) 637

Castillo, C., see Zurita, M.P. (3) 463-473

Cederholm, T., see Hjorth, E. (4) 697-713

Cerami, C., A. Marcone, D. Galimberti, M. Zamboni, C. Fenoglio, M. Serpente, E. Scarpini and S.F.
Cappa, Novel Evidence of Phenotypical Variability in the Hexanucleotide Repeat Expansion in Chromosome 9 (3) 455-462

Chamberlain, D., see Acharya, N.K. (1) 179-198

Chan, W.C., see Lee, A.T.C. (4) 687-696

Chan, W.M., see Lee, A.T.C. (4) 687-696

Chapman, D., see Claxton, A. (4) 789-797

Chau, W.H., see Yuan, Q. (4) 675-685

Chauhan, B.C., see Xiao, C. (4) 777-788

Chauhan, N.B., see Xiao, C. (4) 777-788

Chen, C.L.-H., see Dong, Y. (1) 159-168

Cheng, X.-S., see Jiang, X. (1) 91-105

Chin, J., see Cho, H. (4) 813-821

Chiu, H.F.K., see Lee, A.T.C. (4) 687-696

Cho, H., S.W. Seo, J.-H. Kim, M.K. Suh, J.-H. Lee, Y.S. Choe, K.-H. Lee, J.S. Kim, G.H. Kim, Y. Noh, B.S. Ye, H.J. Kim, C.W. Yoon, J. Chin and D.L. $\mathrm{Na}$, Amyloid Deposition in Early Onset versus Late Onset Alzheimer's Disease (4) 813-821

Choe, Y.S., see Cho, H. (4) 813-821

Cholerton, B., see Claxton, A. (4) 789-797

Cioffi, S., see Villa, C. (3) 487-494

Clarkson, M.J., see Scahill, R.I. (1) 199-212

Claxton, A., L.D. Baker, C.W. Wilkinson, E.H. Trittschuh, D. Chapman, G.S. Watson, B. Cholerton, S.R. Plymate, M. Arbuckle and S. Craft, Sex and ApoE Genotype Differences in Treatment Response to Two Doses of Intranasal Insulin in Adults with Mild Cognitive Impairment or Alzheimer's Disease (4) 789-797

Clerici, F., see Villa, C. (3) 487-494

Clifford, P.M., see Acharya, N.K. (1) 179-198

Confaloni, A., see Piscopo, P. (2) 313-318

Cooper, G.E., see Kryscio, R.J. (4) 823-832

Coretti, N.J., see Acharya, N.K. (1) 179-198

Correia, S.C., see Carvalho, C. (3) 623-635

Craft, S., see Claxton, A. (4) 789-797

Crestini, A., see Piscopo, P. (2) 313-318

Crisi, G., see Mitolo, M. (1) 75-90

Crutch, S.J., see Scahill, R.I. (1) 199-212

da Cruz e Silva, E.F., see Rebelo, S. (4) 761-775

da Cruz e Silva, O.A.B., see Rebelo, S. (4) 761-775

Daborg, J., S. Holmgren, A. Abramsson, U. Andreasson, M. Zetterberg, S. Nilsson, L. Minthon, I. Skoog, K. Blennow, M. Pekna, E. Hanse and H. Zetterberg, Complement Gene Single Nucleotide Polymorphisms and Biomarker Endophenotypes of Alzheimer's Disease (1) 51-57

Dal-Bianco, P., see Benke, T. (2) 247-252

Davis, F.J., see Xiao, C. (4) 777-788 
Dawe, G.S., see Ong, W.-Y. (4) 643-668

De Deyn, P.P., see Engelborghs, S. (1) 67-73

de Leon, M.J., see Glodzik, L. (3) 427-440

de Yebenes, J.G., see Casarejos, M.J. (3) 525-539

del Pozo, F., see Fernández, A. (3) 495-507

del Valle, E., see Navarro, A. (3) 589-597

Delazer, M., see Benke, T. (2) 247-252

Delgado, C., see Slachevsky, A. (2) 297-306

DeMarshall, C., see Acharya, N.K. (1) 179-198

Di Santo, S.G., F. Prinelli, F. Adorni, C. Caltagirone and M. Musicco, A Meta-Analysis of the Efficacy of Donepezil, Rivastigmine, Galantamine, and Memantine in Relation to Severity of Alzheimer's Disease (2) 349-361

Domingues, S.C., see Rebelo, S. (4) 761-775

Don, A., see Bhatia, S. (3) 475-486

Dong, Y., W.S. Pang, L.B.S. Lim, Y.-H. Yang, J.C. Morris, S. Hilal, N. Venketasubramanian and C.L.-H. Chen, The Informant AD8 is Superior to Participant AD8 in Detecting Cognitive Impairment in a Memory Clinic Setting (1) 159-168

Du, L.-L., see Jiang, X. (1) 91-105

Duan, Y., see Han, G. (2) 373-386

Durieu, E., see Hochard, A. (1) 107-120

Edwards, M., see Hall, J.R. (2) 363-371

Elbert, T., see Thurm, F. (3) 575-587

Eldik, L.J.V., see Kryscio, R.J. (4) 823-832

Elias-Sonnenschein, L.S., see Vermeiren, A.P.A. 241-246

Engelborghs, S., K. Sleegers, S. Van der Mussele, N. Le Bastard, N. Brouwers, C. Van Broeckhoven and P.P. De Deyn, Brain-Specific Tryptophan Hydroxylase, TPH2, and 5-HTTLPR are Associated with Frontal Lobe Symptoms in Alzheimer's Disease (1) 67-73

Engelborghs, S., see Slaets, S. (1) 137-146

Ercoli, L.M., see Merrill, D.A. (1) 147-157

Erickson, M.A., see Rhodehouse, B.C. (1) 59-66

Eriksdotter, M., see Hjorth, E. (4) 697-713

Esteves, S.L.C., see Rebelo, S. (4) 761-775

Ewers, M., see Vermeiren, A.P.A. (2) 241-246

Faivre, E., and C. Hölscher, D-Ala ${ }^{2}$ GIP Facilitated Synaptic Plasticity and Reduces Plaque Load in Aged Wild Type Mice and in an Alzheimer's Disease Mouse Model (2) 267-283

Fant, X., see Hochard, A. (1) 107-120

Fardilha, M., see Rebelo, S. (4) 761-775

Fardo, D.W., see Kryscio, R.J. (4) 823-832

Farlow, M.R., see Wang, Y. (4) 751-760

Farooqui, A.A., see Ong, W.-Y. (4) 643-668
Fasano, F., see Mitolo, M. (1) 75-90

Faxen-Irving, G., see Hjorth, E. (4) 697-713

Felicitá, V., see Rispoli, V. (4) 833-846

Feng, X., see Han, G. (2) 373-386

Fenoglio, C., see Cerami, C. (3) 455-462

Fenoglio, C., see Arosio, B. (4) 669-674

Fenoglio, C., see Villa, C. (3) 487-494

Fernández, A., A. Turrero, P. Zuluaga, P. Gil-Gregorio, F. del Pozo, F. Maestu and S. Moratti, MEG Delta Mapping Along the Healthy Aging-Alzheimer's Disease Continuum: Diagnostic Implications (3) 495-507

Fernández, E., see Martinez-Mir, A. (2) 403-412

Fernandez-Estevez, M., see Casarejos, M.J. (3) 525539

Ferrer, I., see Aso, E. (4) 847-858

Ferri, E., see Arosio, B. (4) 669-674

Figueroa-Corona, María del P., see Lira-De León, K.I. (2) 319-334

Flajolet, M., see Hochard, A. (1) 107-120

Fox, N.C., see Scahill, R.I. (1) 199-212

Franceschi, M., see Villa, C. (3) 487-494

Frebourg, T., see Pottier, C. (1) 45-49

Freeman, T., see Acharya, N.K. (1) 179-198

Freund-Levi, Y., see Hjorth, E. (4) 697-713

Frisoni, G.B., see Vermeiren, A.P.A. (2) 241-246

Frölich, L., see Vermeiren, A.P.A. (2) 241-246

Fuentealba, J., see Zurita, M.P. (3) 463-473

Galimberti, D., see Arosio, B. (4) 669-674

Galimberti, D., see Cerami, C. (3) 455-462

Galimberti, D., see Piscopo, P. (2) 313-318

Galimberti, D., see Villa, C. (3) 487-494

Gallone, S., see Villa, C. (3) 487-494

Galons, H., see Hochard, A. (1) 107-120

Gao, S., see Wang, Y. (4) 751-760

García-Gutiérrez, P., see Lira-De León, K.I. (2) 319334

Gardini, S., see Mitolo, M. (1) 75-90

Garner, B., see Bhatia, S. (3) 475-486

Gayán, J., see Martinez-Mir, A. (2) 403-412

Ghezzi, L., see Villa, C. (3) 487-494

Gil-Gregorio, P., see Fernández, A. (3) 495-507

Glodzik, L., C. Randall, H. Rusinek and M.J. de Leon, Cerebrovascular Reactivity to Carbon Dioxide in Alzheimer's Disease (3) 427-440

Gloger, S., see Slachevsky, A. (2) 297-306

Gloulou, O., see Hochard, A. (1) 107-120

Gomez, A., see Casarejos, M.J. (3) 525-539

Gómez, P., see Zurita, M.P. (3) 463-473

González-Pérez, A., see Martinez-Mir, A. (2) 403-412 
Graff, C., see Vermeiren, A.P.A. (2) 241-246

Grave, G., see Roed, L. (3) 611-621

Guan, C., see Acharya, N.K. (1) 179-198

Gussago, C., see Arosio, B. (4) 669-674

Guttin, A., see Nissou, M.-F. (3) 553-564

Guzman, M., see Casarejos, M.J. (3) 525-539

Hagman, G., see Jelic, V. (2) 285-295

Haldar, S., see Singh, A. (3) 541-552

Hall, A., see Muñoz-Ruiz, M.Á. (4) 727-739

Hall, J.R., A.R. Wiechmann, L.A. Johnson, M. Edwards, R.C. Barber, A.S. Winter, M. Singh, S.E. O'Bryant and for the Texas Alzheimer's Research and Care Consortium, Biomarkers of Vascular Risk, Systemic Inflammation, and Microvascular Pathology and Neuropsychiatric Symptoms in Alzheimer's Disease (2) 363-371

Hampel, H., see Vermeiren, A.P.A. (2) 241-246

Han, G., J. Wang, F. Zeng, X. Feng, J. Yu, H.-Y. Cao, X. Yi, H. Zhou, L.-W. Jin, Y. Duan, Y.-J. Wang and H. Lei, Characteristic Transformation of Blood Transcriptome in Alzheimer's Disease (2) 373-386

Han, M., see Acharya, N.K. (1) 179-198

Han, S.-H., see Kim, H.-J. (1) 129-136

Hannequin, D., see Pottier, C. (1) 45-49

Hanse, E., see Daborg, J. (1) 51-57

Hardy, J., see Scahill, R.I. (1) 199-212

Härtig, W., see Wirths, O. (4) 741-749

Hartikainen, P., see Muñoz-Ruiz, M.Á. (4) 727-739

Hatsuta, H., see Wen, Y. (2) 387-394

Hébert, S.S., W.-X. Wang, Q. Zhu and P.T. Nelson, A Study of Small RNAs from Cerebral Neocortex of Pathology-Verified Alzheimer's Disease, Dementia with Lewy Bodies, Hippocampal Sclerosis, Frontotemporal Lobar Dementia, and Non-Demented Human Controls (2) 335-348

Herukka, S.-K., see Muñoz-Ruiz, M.Á. (4) 727-739

Hilal, S., see Dong, Y. (1) 159-168

Hillmann, A., see Wirths, O. (4) 741-749

Hjorth, E., M. Zhu, V.C. Toro, I. Vedin, J. Palmblad, T. Cederholm, Y. Freund-Levi, G. Faxen-Irving, L.-O. Wahlund, H. Basun, M. Eriksdotter and M. Schultzberg, Omega-3 Fatty Acids Enhance Phagocytosis of Alzheimer's Disease-Related Amyloid- $\beta_{42}$ by Human Microglia and Decrease Inflammatory Markers (4) 697-713

Hochard, A., N. Oumata, K. Bettayeb, O. Gloulou, X. Fant, E. Durieu, N. Buron, M. Porceddu, A. BorgneSanchez, H. Galons, M. Flajolet and L. Meijer, Aftins Increase Amyloid- $\beta_{42}$, Lower Amyloid- $\beta_{38}$, and Do Not Alter Amyloid- $\beta_{40}$ Extracellular Production in vitro: Toward a Chemical Model of Alzheimer's Disease? (1) 107-120

Hoeschel, M., see Schmidt, C. (3) 451-454

Holmgren, S., see Daborg, J. (1) 51-57

Hölscher, C., see Faivre, E. (2) 267-283

Horback, K., see Singh, A. (3) 541-552

Horndalsveen, P.O., see Roed, L. (3) 611-621

Hovingh, G.K., see Shahim, P. (2) 307-312

Huang, J.-D., see Yuan, Q. (4) 675-685

Hui, L.Y.F., see Lee, A.T.C. (4) 687-696

Ihara, Y., see Wen, Y. (2) 387-394

Ikonomovic, M.D., see Scheff, S.W. (3) 599-609

Imfeld, P., Y.B.B. Pernus, S.S. Jick and C.R. Meier, Epidemiology, Co-Morbidities, and Medication Use of Patients with Alzheimer's Disease or Vascular Dementia in the UK (3) 565-573

Inglese, S., see Arosio, B. (4) 669-674

Issartel, J.-P., see Nissou, M.-F. (3) 553-564

Ittner, L.M., see Ong, W.-Y. (4) 643-668

Jacobs, H.I.L., see Bruner, E. (2) 227-240

Javitt, N.B., Alzheimer's Disease: Neuroprogesterone, Epoxycholesterol, and ABC Transporters as Determinants of Neurodesmosterol Tissue Levels and its Role in Amyloid Protein Processing (3) 441-450

Jelic, V., G. Hagman, N.G. Yamamoto, Y. Teranishi, T. Nishimura, B. Winblad and P.F. Pavlov, Abnormal Platelet Amyloid- $\beta$ Protein Precursor (A $\beta$ PP) Metabolism in Alzheimer's Disease: Identification and Characterization of a New A $\beta$ PP Isoform as Potential Biomarker (2) 285-295

Jenner, A.M., see Bhatia, S. (3) 475-486

Jia, L.-W., see Jiang, X. (1) 91-105

Jiang, X., L.-W. Jia, X.-H. Li, X.-S. Cheng, J.-Z. Xie, Z.-W. Ma, W.-J. Xu, Y. Liu, Y. Yao, L.-L. Du and X.-W. Zhou, Capsaicin Ameliorates StressInduced Alzheimer's Disease-Like Pathological and Cognitive Impairments in Rats (1) 91-105

Jicha, G.A., see Kryscio, R.J. (4) 823-832

Jick, S.S., see Imfeld, P. (3) 565-573

Jimenez, O., see Slachevsky, A. (2) 297-306

Jin, L.-W., see Han, G. (2) 373-386

Johnson, D.K., see Watts, A.S. (2) 253-265

Johnson, L.A., see Hall, J.R. (2) 363-371

Jones, R.W., see Vermeiren, A.P.A. (2) 241-246

Julkunen, V., see Muñoz-Ruiz, M.Á. (4) 727-739

Juvés, S., see Aso, E. (4) 847-858 
Kain, N., see Bhatia, S. (3) 475-486

Kakita, A., see Wen, Y. (2) 387-394

Kang, J.H., see Vercambre, M.-N. (2) 413-421

Kapeller, P., see Benke, T. (2) 247-252

Karch, A., see Schmidt, C. (3) 451-454

Kehoe, P.G., see Vermeiren, A.P.A. (2) 241-246

Kepe, V., see Merrill, D.A. (1) 147-157

Kim, G.H., see Cho, H. (4) 813-821

Kim, H.J., see Cho, H. (4) 813-821

Kim, H.-J., W.-J. Moon and S.-H. Han, Differential Cholinergic Pathway Involvement in Alzheimer's Disease and Subcortical Ischemic Vascular Dementia (1) 129-136

Kim, J.-H., see Cho, H. (4) 813-821

Kim, J.S., see Cho, H. (4) 813-821

Kitamura, N., see Wen, Y. (2) 387-394

Klein, W.L., see Xiao, C. (4) 777-788

Koikkalainen, J., see Muñoz-Ruiz, M.Á. (4) 727-739

Kolassa, I.-T., see Thurm, F. (3) 575-587

Kolassa, S., see Thurm, F. (3) 575-587

Kosaka, K., see Wen, Y. (2) 387-394

Kosciuk, M.C., see Acharya, N.K. (1) 179-198

Kril, J.J., see Bhatia, S. (3) 475-486

Kryscio, R.J., E.L. Abner, Y. Lin, G.E. Cooper, D.W. Fardo, G.A. Jicha, P.T. Nelson, C.D. Smith, L.J.V. Eldik, L. Wan and F.A. Schmitt, Adjusting for Mortality when Identifying Risk Factors for Transitions to Mild Cognitive Impairment and Dementia (4) 823-832

Kuwano, R., see Wen, Y. (2) 387-394

Lacor, P.N., see Xiao, C. (4) 777-788

Lam, L.C.W., see Lee, A.T.C. (4) 687-696

Lannfelt, L., see Roed, L. (3) 611-621

Lautner, R., see Shahim, P. (2) 307-312

Lavretsky, H., see Merrill, D.A. (1) 147-157

Le Bastard, N., see Engelborghs, S. (1) 67-73

Leblhuber, F., see Benke, T. (2) 247-252

Lee, A.T.C., W.C. Chan, H.F.K. Chiu, M. Richards, S.P.S. Ng, L.Y.F. Hui, W.M. Chan and L.C.W. Lam, Widened Pulse Pressure is a Potential Risk Factor for Significant Cognitive Impairment among Community-Dwelling Chinese Younger Old People (4) 687-696

Lee, J.-H., see Cho, H. (4) 813-821

Lee, K.-H., see Cho, H. (4) 813-821

Lee, S., J. Zemianek and T.B. Shea, Rapid, Reversible Impairment of Synaptic Signaling in Cultured Cortical Neurons by Exogenously-Applied Amyloid- $\beta$ (2) 395-402

Leenheir, E.D., see Slaets, S. (1) 137-146
Lei, H., see Han, G. (2) 373-386

Lenoir, H., see Vermeiren, A.P.A. (2) 241-246

Leo, A., see Rispoli, V. (4) 833-846

Levin, E.C., see Acharya, N.K. (1) 179-198

Li, H., see Bhatia, S. (3) 475-486

Li, X.-H., see Jiang, X. (1) 91-105

Lim, L.B.S., see Dong, Y. (1) 159-168

Lin, Y., see Kryscio, R.J. (4) 823-832

Lin, Z.-X., see Yuan, Q. (4) 675-685

Lindahl, T., see Roed, L. (3) 611-621

Lira-De León, K.I., P. García-Gutiérrez, I.N. Serratos, M. Palomera-Cárdenas, María del P. FigueroaCorona, V. Campos-Peña and M.A. Meraz-Ríos, Molecular Mechanism of Tau Aggregation Induced by Anionic and Cationic Dyes (2) 319-334

Liu, Y., see Jiang, X. (1) 91-105

Liu, Y., see Muñoz-Ruiz, M.Á. (4) 727-739

Lladó, A., see Arenaza-Urquijo, E.M. (4) 715-726

Lönneborg, A., see Roed, L. (3) 611-621

Lopez-Arrieta, J.M., see Martinez-Mir, A. (2) 403-412

Loskutova, N., see Watts, A.S. (2) 253-265

Lötjönen, J., see Muñoz-Ruiz, M.Á. (4) 727-739

Luyckx, J., see Slaets, S. (1) 137-146

Ma, Z.-W., see Jiang, X. (1) 91-105

Machado, N., see Carvalho, C. (3) 623-635

Macphee, C.H., see Acharya, N.K. (1) 179-198

Macreadie, I.G., see Porzoor, A. (2) 217-225

Maestu, F., see Fernández, A. (3) 495-507

MaGee, T.R., see Wang, Y. (4) 751-760

Maldonado, R., see Aso, E. (4) 847-858

Marcone, A., see Cerami, C. (3) 455-462

Marcone, A., see Villa, C. (3) 487-494

Mari, D., see Arosio, B. (4) 669-674

Mariani, C., see Villa, C. (3) 487-494

Marín, J., see Martinez-Mir, A. (2) 403-412

Marksteiner, J., see Benke, T. (2) 247-252

Marra, R., see Rispoli, V. (4) 833-846

Martin, J.-J., see Slaets, S. (1) 137-146

Mártínez, E., see Navarro, A. (3) 589-597

Martinez-Mir, A., A. González-Pérez, J. Gayán, C. Antúnez, J. Marín, M. Boada, J.M. Lopez-Arrieta, Alzheimer's Disease Neuroimaging Initiative, E. Fernández, R. Ramírez-Lorca, M.E. Sáez, A. Ruiz, F.G. Scholl and L.M. Real, Genetic Study of Neurexin and Neuroligin Genes in Alzheimer's Disease (2) 403-412

Martorell, B., see Slachevsky, A. (2) 297-306

Matthews, D.C., see Mosconi, L. (3) 509-524

Mattila, J., see Muñoz-Ruiz, M.Á. (4) 727-739

Mattsson, N., see Shahim, P. (2) 307-312 
McDonald, B.C., see Wang, Y. (4) 751-760

Mead, S., see Scahill, R.I. (1) 199-212

Meier, C.R., see Imfeld, P. (3) 565-573

Meijer, L., see Hochard, A. (1) 107-120

Mena, M.A., see Casarejos, M.J. (3) 525-539

Meraz-Ríos, M.A., see Lira-De León, K.I. (2) 319-334

Merrill, D.A., P. Siddarth, V. Kepe, P.V. Raja, N. Saito, L.M. Ercoli, K.J. Miller, H. Lavretsky, S.Y. Bookheimer, J.R. Barrio and G.W. Small, Vascular Risk and FDDNP-PET Influence Cognitive Performance (1) 147-157

Meyerson, H., see Singh, A. (3) 541-552

Miller, K.J., see Merrill, D.A. (1) 147-157

Minthon, L., see Daborg, J. (1) 51-57

Minthon, L., see Sjölander, A. (1) 121-127

Minthon, L., see Vermeiren, A.P.A. (2) 241-246

Miranda-Castillo, C., see Slachevsky, A. (2) 297-306

Mitolo, M., S. Gardini, F. Fasano, G. Crisi, A. Pelosi, F. Pazzaglia and P. Caffarra, Visuospatial Memory and Neuroimaging Correlates in Mild Cognitive Impairment (1) 75-90

Miyashita, A., see Wen, Y. (2) 387-394

Molinuevo, J.-L., see Arenaza-Urquijo, E.M. (4) 715-726

Moon, W.-J., see Kim, H.-J. (1) 129-136

Moratti, S., see Fernández, A. (3) 495-507

Moreira, P.I., see Carvalho, C. (3) 623-635

Morris, J.C., see Dong, Y. (1) 159-168

Morris, J.C., see Shim, Y.S. (4) 799-811

Mosconi, L., R.D. Andrews, D.C. Matthews and For the Alzheimer's Disease Neuroimaging Initiative (ADNI), Comparing Brain Amyloid Deposition, Glucose Metabolism, and Atrophy in Mild Cognitive Impairment with and without a Family History of Dementia (3) 509-524

Mota, P.C., see Carvalho, C. (3) 623-635

Motazacker, M.M., see Shahim, P. (2) 307-312

Mufson, E.J., see Scheff, S.W. (3) 599-609

Muñoz, G., see Zurita, M.P. (3) 463-473

Muñoz, M.P., see Casarejos, M.J. (3) 525-539

Muñoz-Neira, C., see Slachevsky, A. (2) 297-306

Muñoz-Ruiz, M.Á., P. Hartikainen, A. Hall, J. Mattila, J. Koikkalainen, S.-K. Herukka, V. Julkunen, R. Vanninen, Y. Liu, J. Lötjönen and H. Soininen, Disease State Fingerprint in Frontotemporal Degeneration with Reference to Alzheimer's Disease and Mild Cognitive Impairment (4) 727-739

Murayama, S., see Wen, Y. (2) 387-394

Musicco, M., see Di Santo, S.G. (2) 349-361

Na, D.L., see Cho, H. (4) 813-821

Nagele, E.P., see Acharya, N.K. (1) 179-198
Nagele, R.G., see Acharya, N.K. (1) 179-198

Navarro, A., E. del Valle, E. Mártínez, C. Ordóñez, C. Pérez and J. Tolivia, Highly Selective and Fast Diagnosis of Alzheimer's Disease Hallmark Lesions using Congo Red in Isopropyl Alcoholic Solution (3) 589-597

Nelson, P.T., see Hébert, S.S. (2) 335-348

Nelson, P.T., see Kryscio, R.J. (4) 823-832

Ng, C.T., see Yuan, Q. (4) 675-685

Ng, S.P.S., see Lee, A.T.C. (4) 687-696

Nilsson, C., see Roed, L. (3) 611-621

Nilsson, S., see Daborg, J. (1) 51-57

Nilsson, S., see Sjölander, A. (1) 121-127

Nishimura, T., see Jelic, V. (2) 285-295

Nissou, M.-F., J. Brocard, M.E. Atifi, A. Guttin, A. Andrieux, F. Berger, J.-P. Issartel and D. Wion, The Transcriptomic Response of Mixed NeuronGlial Cell Cultures to 1,25-Dihydroxyvitamin D3 Includes Genes Limiting the Progression of Neurodegenerative Diseases (3) 553-564

Nisticò, R., see Rispoli, V. (4) 833-846

Nobili, F.M., see Vermeiren, A.P.A. (2) 241-246

Noh, Y., see Cho, H. (4) 813-821

Núñez-Huasaf, J., see Slachevsky, A. (2) 297-306

Nuytinck, L., see Sjölander, A. (1) 121-127

O’Bryant, S.E., see Hall, J.R. (2) 363-371

O’Neill, D.P., see Wang, Y. (4) 751-760

Oliveira, C.R., see Carvalho, C. (3) 623-635

Olives, J., see Arenaza-Urquijo, E.M. (4) 715-726

Ong, W.-Y., K. Tanaka, G.S. Dawe, L.M. Ittner and A.A. Farooqui, Slow Excitotoxicity in Alzheimer's Disease (4) 643-668

Opazo, C., see Zurita, M.P. (3) 463-473

Ordóñez, C., see Navarro, A. (3) 589-597

Oumata, N., see Hochard, A. (1) 107-120

Ourselin, S., see Scahill, R.I. (1) 199-212

Palmblad, J., see Hjorth, E. (4) 697-713

Palomera-Cárdenas, M., see Lira-De León, K.I. (2) 319-334

Pang, W.S., see Dong, Y. (1) 159-168

Pavlov, P.F., see Jelic, V. (2) 285-295

Pazzaglia, F., see Mitolo, M. (1) 75-90

Pekna, M., see Daborg, J. (1) 51-57

Pelosi, A., see Mitolo, M. (1) 75-90

Pérez, C., see Navarro, A. (3) 589-597

Pernus, Y.B.B., see Imfeld, P. (3) 565-573

Perry, G., R. Rodrigues and R.J. Castellani, Are Alzheimer's Disease and Aging Evolutionary? (3) 637 
Perucho, J., see Casarejos, M.J. (3) 525-539

Piscopo, P., R. Rivabene, D. Galimberti, A. Crestini, G. Talarico, N. Vanacore, E. Scarpini, G. Bruno and A. Confaloni, Gender Effects on Plasma PGRN Levels in Patients with Alzheimer's Disease: A Preliminary Study (2) 313-318

Plymate, S.R., see Claxton, A. (4) 789-797

Pollaro, M., see Acharya, N.K. (1) 179-198

Porceddu, M., see Hochard, A. (1) 107-120

Porzoor, A., and I.G. Macreadie, Application of Yeast to Study the Tau and Amyloid- $\beta$ Abnormalities of Alzheimer's Disease (2) 217-225

Pottier, C., D. Wallon, S. Rousseau, A. Rovelet-Lecrux, A.-C. Richard, A. Rollin-Sillaire, T. Frebourg, D. Campion, D. Hannequin and GMAJ/COMAJ collaborators, TREM2 R47H Variant as a Risk Factor for Early-Onset Alzheimer's Disease (1) 45-49

Pradier, L., see Wirths, O. (4) 741-749

Price, D.A., see Scheff, S.W. (3) 599-609

Prinelli, F., see Di Santo, S.G. (2) 349-361

Prins, N.D., see van der Vlies, A.E. (1) 169-178

Ragusa, S., see Rispoli, V. (4) 833-846

Rainero, I., see Villa, C. (3) 487-494

Raja, P.V., see Merrill, D.A. (1) 147-157

Rami, L., see Arenaza-Urquijo, E.M. (4) 715-726

Ramírez-Lorca, R., see Martinez-Mir, A. (2) 403-412

Randall, C., see Glodzik, L. (3) 427-440

Ransmayr, G., see Benke, T. (2) 247-252

Real, L.M., see Martinez-Mir, A. (2) 403-412

Rebelo, S., S.C. Domingues, M. Santos, M. Fardilha, S.L.C. Esteves, S.I. Vieira, A.P.B. Vintém, W. Wu, E.F. da Cruz e Silva and O.A.B. da Cruz e Silva, Identification of a Novel Complex A $\beta P P: F e 65: P P 1$ that Regulates A $\beta P P \mathrm{Thr}^{668}$ Phosphorylation Levels (4) 761-775

Reiber, J.H.C., see van der Vlies, A.E. (1) 169-178

Rhodehouse, B.C., M.A. Erickson, W.A. Banks and S.E. Bearden, Hyperhomocysteinemic Mice Show Cognitive Impairment Without Features of Alzheimer's Disease Phenotype (1) 59-66

Rian, E., see Roed, L. (3) 611-621

Richard, A.-C., see Pottier, C. (1) 45-49

Richards, M., see Lee, A.T.C. (4) 687-696

Ridgway, G.R., see Scahill, R.I. (1) 199-212

Ridolfi, E., see Arosio, B. (4) 669-674

Ridolfi, E., see Villa, C. (3) 487-494

Rigaud, A.-S., see Vermeiren, A.P.A. (2) 241-246

Rikkert, M.O., see Vermeiren, A.P.A. (2) 241-246

Risacher, S.L., see Wang, Y. (4) 751-760
Rispoli, V., S. Ragusa, R. Nisticò, R. Marra, E. Russo, A. Leo, V. Felicitá and D. Rotiroti, Huperzine A Restores Cortico-Hippocampal Functional Connectivity after Bilateral AMPA Lesion of the Nucleus Basalis of Meynert (4) 833-846

Ritchie, K., see Vercambre, M.-N. (2) 413-421

Rivabene, R., see Piscopo, P. (2) 313-318

Roberts, K.N., see Scheff, S.W. (3) 599-609

Rodrigues, R., see Perry, G. (3) 637

Roe, C.M., see Shim, Y.S. (4) 799-811

Roed, L., G. Grave, T. Lindahl, E. Rian, P.O. Horndalsveen, L. Lannfelt, C. Nilsson, F. Swenson, A. Lönneborg, P. Sharma and M. Sjögren, Prediction of Mild Cognitive Impairment that Evolves into Alzheimer's Disease Dementia within Two Years using a Gene Expression Signature in Blood: A Pilot Study (3) 611-621

Rollin-Sillaire, A., see Pottier, C. (1) 45-49

Rossi, P.D., see Arosio, B. (4) 669-674

Rossor, M.N., see Scahill, R.I. (1) 199-212

Rotiroti, D., see Rispoli, V. (4) 833-846

Rousseau, S., see Pottier, C. (1) 45-49

Rovelet-Lecrux, A., see Pottier, C. (1) 45-49

Ruberu, K., see Bhatia, S. (3) 475-486

Ruiz, A., see Martinez-Mir, A. (2) 403-412

Ruiz, J.F., see Casarejos, M.J. (3) 525-539

Rusinek, H., see Glodzik, L. (3) 427-440

Russo, E., see Rispoli, V. (4) 833-846

Ryan, N.S., see Scahill, R.I. (1) 199-212

Sáez, M.E., see Martinez-Mir, A. (2) 403-412

Sagredo, O., see Casarejos, M.J. (3) 525-539

Saito, N., see Merrill, D.A. (1) 147-157

Saito, Y., see Wen, Y. (2) 387-394

Sala-Llonch, R., see Arenaza-Urquijo, E.M. (4) 715726

Sánchez-Valle, R., see Arenaza-Urquijo, E.M. (4) 715726

Sanin, G., see Benke, T. (2) 247-252

Santos, M., see Rebelo, S. (4) 761-775

Santos, M.S., see Carvalho, C. (3) 623-635

Santos, R.X., see Carvalho, C. (3) 623-635

Saykin, A.J., see Wang, Y. (4) 751-760

Scahill, R.I., G.R. Ridgway, J.W. Bartlett, J. Barnes, N.S. Ryan, S. Mead, J. Beck, M.J. Clarkson, S.J. Crutch, J.M. Schott, S. Ourselin, J.D. Warren, J. Hardy, M.N. Rossor and N.C. Fox, Genetic Influences on Atrophy Patterns in Familial Alzheimer's Disease: A Comparison of APP and PSEN1 Mutations (1) 199-212

Scarpini, E., see Cerami, C. (3) 455-462 
Scarpini, E., see Arosio, B. (4) 669-674

Scarpini, E., see Piscopo, P. (2) 313-318

Scarpini, E., see Villa, C. (3) 487-494

Scheff, S.W., D.A. Price, F.A. Schmitt, K.N. Roberts, M.D. Ikonomovic and E.J. Mufson, Synapse Stability in the Precuneus Early in the Progression of Alzheimer's Disease (3) 599-609

Scheltens, P., see van der Vlies, A.E. (1) 169-178

Scheltens, P., see Vermeiren, A.P.A. (2) 241-246

Schlee, W., see Thurm, F. (3) 575-587

Schmidt, C., A. Karch, S. Artjomova, M. Hoeschel and I. Zerr, Pre-Progression Rates in Alzheimer's Disease Revisited (3) 451-454

Schmidt, H., see Benke, T. (2) 247-252

Schmidt, R., see Benke, T. (2) 247-252

Schmitt, F.A., see Kryscio, R.J. (4) 823-832

Schmitt, F.A., see Scheff, S.W. (3) 599-609

Scholl, F.G., see Martinez-Mir, A. (2) 403-412

Schott, J.M., see Scahill, R.I. (1) 199-212

Schultzberg, M., see Hjorth, E. (4) 697-713

Seiler, S., see Benke, T. (2) 247-252

Seo, S.W., see Cho, H. (4) 813-821

Sepúlveda, F.J., see Zurita, M.P. (3) 463-473

Serpente, M., see Arosio, B. (4) 669-674

Serpente, M., see Cerami, C. (3) 455-462

Serpente, M., see Villa, C. (3) 487-494

Serratos, I.N., see Lira-De León, K.I. (2) 319-334

Shahim, P., A.E. Bochem, N. Mattsson, R. Lautner, K. Blennow, G.K. Hovingh, M.M. Motazacker and H. Zetterberg, Plasma Amyloid- $\beta$ in Patients with Tangier Disease (2) 307-312

Sharma, P., see Roed, L. (3) 611-621

Shea, T.B., see Lee, S. (2) 395-402

Shepherd, C.E., see Bhatia, S. (3) 475-486

Shi, Y., see Acharya, N.K. (1) 179-198

Shim, Y.S., C.M. Roe, V.D. Buckles and J.C. Morris, Clinicopathologic Study of Alzheimer's Disease: Alzheimer Mimics (4) 799-811

Siddarth, P., see Merrill, D.A. (1) 147-157

Silva, J.R., see Slachevsky, A. (2) 297-306

Singh, A., S. Haldar, K. Horback, C. Tom, L. Zhou, H. Meyerson and N. Singh, Prion Protein Regulates Iron Transport by Functioning as a Ferrireductase (3) 541-552

Singh, M., see Hall, J.R. (2) 363-371

Singh, N., see Singh, A. (3) 541-552

Sjögren, M., see Roed, L. (3) 611-621

Sjölander, A., L. Minthon, L. Nuytinck, E. Vanmechelen, K. Blennow and S. Nilsson, Functional MannoseBinding Lectin Haplotype Variants are Associated with Alzheimer's Disease (1) 121-127
Skoog, I., see Daborg, J. (1) 51-57

Slachevsky, A., M. Budinich, C. Miranda-Castillo, J. Núñez-Huasaf, J.R. Silva, C. Muñoz-Neira, S. Gloger, O. Jimenez, B. Martorell and C. Delgado, The CUIDEME Study: Determinants of Burden in Chilean Primary Caregivers of Patients with Dementia (2) 297-306

Slaets, S., N.L. Bastard, J. Theuns, K. Sleegers, A. Verstraeten, E.D. Leenheir, J. Luyckx, J.-J. Martin, C.V. Broeckhoven and S. Engelborghs, Amyloid Pathology Influences $A \beta_{1-42}$ Cerebrospinal Fluid Levels in Dementia with Lewy Bodies (1) 137-146

Sleegers, K., see Engelborghs, S. (1) 67-73

Sleegers, K., see Slaets, S. (1) 137-146

Small, G.W., see Merrill, D.A. (1) 147-157

Smith, C.D., see Kryscio, R.J. (4) 823-832

Soininen, H., see Muñoz-Ruiz, M.Á. (4) 727-739

Soininen, H., see Vermeiren, A.P.A. (2) 241-246

Solé-Padullés, C., see Arenaza-Urquijo, E.M. (4) 715 726

Song, Y.-Q., see Yuan, Q. (4) 675-685

Spiro, A.S., see Bhatia, S. (3) 475-486

Spiru, L., see Vermeiren, A.P.A. (2) 241-246

Staekenborg, S.S., see van der Vlies, A.E. (1) 169-178

Su, H., see Yuan, Q. (4) 675-685

Suh, M.K., see Cho, H. (4) 813-821

Swenson, F., see Roed, L. (3) 611-621

Takahashi, H., see Wen, Y. (2) 387-394

Talarico, G., see Piscopo, P. (2) 313-318

Tanaka, K., see Ong, W.-Y. (4) 643-668

Tedone, E., see Arosio, B. (4) 669-674

Teranishi, Y., see Jelic, V. (2) 285-295

Theuns, J., see Slaets, S. (1) 137-146

Thurm, F., D. Antonenko, W. Schlee, S. Kolassa, T. Elbert and I.-T. Kolassa, Effects of Aging and Mild Cognitive Impairment on Electrophysiological Correlates of Performance Monitoring (3) 575-587

Tolivia, J., see Navarro, A. (3) 589-597

Tom, C., see Singh, A. (3) 541-552

Toro, V.C., see Hjorth, E. (4) 697-713

Tourtellotte, R., see Acharya, N.K. (1) 179-198

Trittschuh, E.H., see Claxton, A. (4) 789-797

Tsolaki, M., see Vermeiren, A.P.A. (2) 241-246

Tsukie, T., see Wen, Y. (2) 387-394

Turrero, A., see Fernández, A. (3) 495-507

Uranüs, M., see Benke, T. (2) 247-252

Van Broeckhoven, C., see Engelborghs, S. (1) 67-73

van de Haar, H.J., see Burgmans, S. (4) 859-873 
van der Flier, W.M., see van der Vlies, A.E. (1) 169-178 Van der Mussele, S., see Engelborghs, S. (1) 67-73 van der Vlies, A.E., S.S. Staekenborg, F. AdmiraalBehloul, N.D. Prins, F. Barkhof, H. Vrenken, J.H.C. Reiber, P. Scheltens and W.M. van der Flier, Associations between Magnetic Resonance Imaging Measures and Neuropsychological Impairment in Early and Late Onset Alzheimer's Disease (1) 169-178

Vanacore, N., see Piscopo, P. (2) 313-318

Vanmechelen, E., see Sjölander, A. (1) 121-127

Vanninen, R., see Muñoz-Ruiz, M.Á. (4) 727-739

Vedin, I., see Hjorth, E. (4) 697-713

Velasco, P.T., see Xiao, C. (4) 777-788

Vellas, B., see Vermeiren, A.P.A. (2) 241-246

Venketasubramanian, N., see Dong, Y. (1) 159-168

Vercambre, M.-N., C. Berr, K. Ritchie and J.H. Kang, Caffeine and Cognitive Decline in Elderly Women at High Vascular Risk (2) 413-421

Verhey, F.R.J., see Burgmans, S. (4) 859-873

Verhey, F.R.J., see Vermeiren, A.P.A. (2) 241-246

Vermeiren, A.P.A., H. Bosma, P.-J. Visser, M.P. Zeegers, C. Graff, M. Ewers, G.B. Frisoni, L. Frölich, H. Hampel, R.W. Jones, P.G. Kehoe, H. Lenoir, L. Minthon, F.M. Nobili, M.O. Rikkert, A.-S. Rigaud, P. Scheltens, H. Soininen, L. Spiru, M. Tsolaki, L.-O. Wahlund, B. Vellas, G. Wilcock, L.S. Elias-Sonnenschein and F.R.J. Verhey, The Association Between APOE $\varepsilon 4$ and Alzheimertype Dementia Among Memory Clinic Patients is Confined to those with a Higher Education. The DESCRIPA Study (2) 241-246

Verstraeten, A., see Slaets, S. (1) 137-146

Vieira, S.I., see Rebelo, S. (4) 761-775

Villa, C., E. Ridolfi, C. Fenoglio, L. Ghezzi, R. Vimercati, F. Clerici, A. Marcone, S. Gallone, M. Serpente, C. Cantoni, R. Bonsi, S. Cioffi, S. Cappa, M. Franceschi, I. Rainero, C. Mariani, E. Scarpini and D. Galimberti, Expression of the Transcription Factor Spl and its Regulatory hsa-miR-29b in Peripheral Blood Mononuclear Cells from Patients with Alzheimer's Disease (3) 487-494

Vimercati, R., see Villa, C. (3) 487-494

Vintém, A.P.B., see Rebelo, S. (4) 761-775

Viola, K.L., see Xiao, C. (4) 777-788

Visser, P.-J., see Vermeiren, A.P.A. (2) 241-246

Vrenken, H., see van der Vlies, A.E. (1) 169-178

Wahlund, L.-O., see Hjorth, E. (4) 697-713

Wahlund, L.-O., see Vermeiren, A.P.A. (2) 241-246
Wallon, D., see Pottier, C. (1) 45-49

Walton, J.R., Aluminum's Involvement in the Progression of Alzheimer's Disease (1) 7-43

Wan, L., see Kryscio, R.J. (4) 823-832

Wang, J., see Han, G. (2) 373-386

Wang, W.-X., see Hébert, S.S. (2) 335-348

Wang, Y., S.L. Risacher, J.D. West, B.C. McDonald, T.R. MaGee, M.R. Farlow, S. Gao, D.P. O’Neill and A.J. Saykin, Altered Default Mode Network Connectivity in Older Adults with Cognitive Complaints and Amnestic Mild Cognitive Impairment (4) 751-760

Wang, Y.-J., see Han, G. (2) 373-386

Warren, J.D., see Scahill, R.I. (1) 199-212

Watson, G.S., see Claxton, A. (4) 789-797

Watts, A.S., N. Loskutova, J.M. Burns and D.K. Johnson, Metabolic Syndrome and Cognitive Decline in Early Alzheimer's Disease and Healthy Older Adults (2) 253-265

Wen, Y., A. Miyashita, N. Kitamura, T. Tsukie, Y. Saito, H. Hatsuta, S. Murayama, A. Kakita, H. Takahashi, H. Akatsu, T. Yamamoto, K. Kosaka, H. Yamaguchi, K. Akazawa, Y. Ihara, R. Kuwano and Japanese Alzheimer's Disease Neuroimaging Initiative, SORL1 is Genetically Associated with Neuropathologically Characterized Late-Onset Alzheimer's Disease (2) 387-394

West, J.D., see Wang, Y. (4) 751-760

Wiechmann, A.R., see Hall, J.R. (2) 363-371

Wilcock, G., see Vermeiren, A.P.A. (2) 241-246

Wilensky, R.L., see Acharya, N.K. (1) 179-198

Wilkinson, C.W., see Claxton, A. (4) 789-797

Winblad, B., see Jelic, V. (2) 285-295

Winter, A.S., see Hall, J.R. (2) 363-371

Wion, D., see Nissou, M.-F. (3) 553-564

Wirths, O., A. Hillmann, L. Pradier, W. Härtig and T.A. Bayer, Oligomeric Pyroglutamate Amyloid- $\beta$ is Present in Microglia and a Subfraction of Vessels in Patients with Alzheimer's Disease: Implications for Immunotherapy (4) 741-749

Wu, W., see Rebelo, S. (4) 761-775

Wu, W., see Yuan, Q. (4) 675-685

Xiao, C., F.J. Davis, B.C. Chauhan, K.L. Viola, P.N. Lacor, P.T. Velasco, W.L. Klein and N.B. Chauhan, Brain Transit and Ameliorative Effects of Intranasally Delivered Anti-Amyloid- $\beta$ Oligomer Antibody in 5XFAD Mice (4) 777-788

Xie, J.-Z., see Jiang, X. (1) 91-105

Xu, W.-J., see Jiang, X. (1) 91-105 
Yamaguchi, H., see Wen, Y. (2) 387-394

Yamamoto, N.G., see Jelic, V. (2) 285-295

Yamamoto, T., see Wen, Y. (2) 387-394

Yang, Y.-H., see Dong, Y. (1) 159-168

Yao, Y., see Jiang, X. (1) 91-105

Ye, B.S., see Cho, H. (4) 813-821

Yi, X., see Han, G. (2) 373-386

Yoon, C.W., see Cho, H. (4) 813-821

Yu, J., see Han, G. (2) 373-386

Yuan, Q., H. Su, Y. Zhang, W.H. Chau, C.T. Ng, Y.-Q.

Song, J.-D. Huang, W. Wu and Z.-X. Lin, Amyloid

Pathology in Spinal Cord of the Transgenic

Alzheimer's Disease Mice is Correlated to the

Corticospinal Tract Pathway (4) 675-685

Zamboni, M., see Cerami, C. (3) 455-462

Zeegers, M.P., see Vermeiren, A.P.A. (2) 241-246

Zemianek, J., see Lee, S. (2) 395-402
Zeng, F., see Han, G. (2) 373-386

Zerr, I., see Schmidt, C. (3) 451-454

Zetterberg, H., see Daborg, J. (1) 51-57

Zetterberg, H., see Shahim, P. (2) 307-312

Zetterberg, M., see Daborg, J. (1) 51-57

Zhang, Y., see Yuan, Q. (4) 675-685

Zhou, H., see Han, G. (2) 373-386

Zhou, L., see Singh, A. (3) 541-552

Zhou, X.-W., see Jiang, X. (1) 91-105

Zhu, M., see Hjorth, E. (4) 697-713

Zhu, Q., see Hébert, S.S. (2) 335-348

Zuluaga, P., see Fernández, A. (3) 495-507

Zurita, M.P., G. Muñoz, F.J. Sepúlveda, P. Gómez, C. Castillo, C.F. Burgos, J. Fuentealba, C. Opazo and L.G. Aguayo, Ibuprofen Inhibits the Synaptic Failure Induced by the Amyloid- $\beta$ Peptide in Hippocampal Neurons (3) 463-473 